\title{
The micropattern effect and visible persistence
}

\author{
E. WILLIAM YUND, HERMAN MORGAN, and ROBERT EFRON \\ Veterans Administration Medical Center, Martinez, California
}

\begin{abstract}
Differences in the temporal order of red and green components within stimuli whose total duration is less than 20 msec may be perceived and detected as color differences-the "micropattern effect." Such brief stimuli also have an extended "visible persistence" in that they appear to last for over $100 \mathrm{msec}$. Although the micropattern effect and visible persistence seem to be operating on different time scales (a few vs. $100 \mathrm{msec}$ ), we suggest an explanation for the micropattern effect in terms of visible persistence. Experiments using micropattern stimuli repeated with various interstimulus intervals (ISIs) support this explanation: The micropattern effect does not reach its full magnitude until the ISI is long enough to accommodate the full duration of the visible persistence.
\end{abstract}

The term "micropattern" was coined by Efron (1973) to refer to a stimulus that has internal variation over time but is so brief that no corresponding variation over time occurs in the percept. An example of such a micropattern is a 20 -msec visual stimulus composed of a $10-\mathrm{msec}$ red light followed by a 10 msec green light presented on the same retinal area. While such a red-green micropattern is too brief for the subject to perceive, the temporal order of the stimulus elements as such (a red light followed by a green light), the temporal structure does affect the percept: the hue of the trailing stimulus element tends to dominate the color. That is to say, if a subject is asked to compare the above red-green micropattern with an otherwise identical green-red micropattern (stimuli that are identical except for the internal temporal order of their elements), he reports that these stimuli have very different colors: the red-green micropattern has a much more greenish- or less reddish-yellow color than the green-red micropattern. Indeed, as little as $1-\mathrm{msec}$ asynchrony between the onsets of the red and green elements of the micropattern is enough to produce near $100 \%$ discrimination between red-green and green-red micropatterns (e.g., see Figure 4A, Yund \& Efron, 1974).

The term "visible persistence" refers to the continuation of the perception of a stimulus after its physical of fset (see Coltheart, 1980, and Long, 1980, for reviews). For relatively long-duration stimuli, visible persistence seems to represent merely the offset latency. However, when the stimulus duration falls below some value (approximately $150 \mathrm{msec}$ at moderate photopic intensities), the situation changes and the shorter the stimulus duration, the longer the

This research was supported by the Medical Research Service of the Veterans Administration. The authors' mailing address is: Neurophysiology-Biophysics Research Laboratory, VA Medical Center, 150 Muir Road, Martinez, California 94553. visible persistence. As described by Efron (1970a, $1970 \mathrm{~b}, 1970 \mathrm{c})$, it is as if there were a minimum duration for a percept, and when the stimulus falls short of that duration, the visible persistence is extended to make up the difference. In other words, it is as if the percept were initiated by stimulus onset (with some latency) and then lasted for some minimum duration unless extended by the continued presence of the stimulus.

Although the micropattern effect and visible persistence may seem to be quite unrelated, the purpose of the present experiment is to test a simple model in which the micropattern effect occurs as a consequence of the phenomenon of visible persistence. The model is illustrated in Figure 1. The upper part of the figure represents a typical visual micropattern stimulus; the lower part represents a possible neural

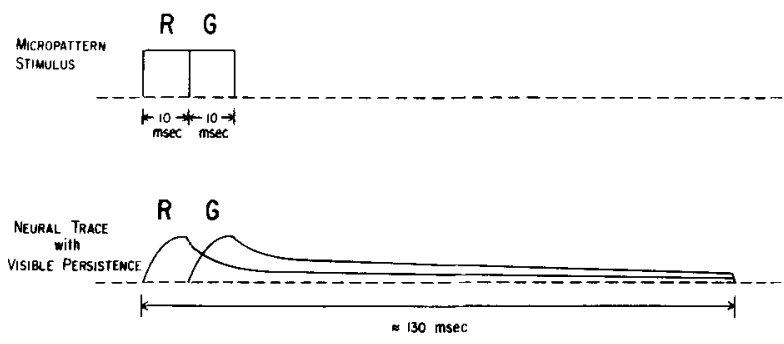

Figure 1. The visible persistence model for the micropattern effect. The upper line of the figure illustrates a typical micropattern stimulus: 10 msec of red light followed immediately by 10 msec of green light. The hue of the trailing stimulus element tends to dominate the perceived color. The lower line of the figure illustrates postulated neural traces for the two stimulus elements, including the temporal extension of these traces representing visible persistence. The 130-msec duration was taken from Efron (1970a, 1970b, 1970c). The precise shape of these traces is not important, but it is important that throughout most of the duration of the neural traces, the trace corresponding to the trailing element has the greater magnitude. The neural traces are illustrated to occur with zero latency for purposes of clarity. 
trace for this stimulus at some level of visual processing after visible persistence has been introduced.

The essence of the model is that the perceived color of the micropattern is derived from the temporal integration of the red-green alternation at the beginning of the neural trace with the much longer part of the trace produced by the visible persistence mechanism. The hue of the trailing stimulus element dominates because the neural trace from the trailing stimulus element has a greater magnitude than that of the leading stimulus element throughout the prolonged interval of the visible persistence. This explanation for the micropattern effect in terms of visible persistence leads to at least one easily testable prediction: If visible persistence is critical to the micropattern effect, then another stimulus presented before the persistence has run its full course should reduce the magnitude of the micropattern effect. That is, we can present the micropattern repetitively and determine how the magnitude of the micropattern effect varies with the rate of repetition-the interstimulus interval (ISI). When the ISI is such that each micropattern encroaches on the persistence of the previous micropattern, the magnitude of micropattern effect should be reduced in proportion to that encroachment. However, once the ISI is longer than the duration of the visible persistence, the micropattern effect should occur at its full magnitude. Since the duration of visible persistence for the stimulus conditions used in these experiments is between 100 and $200 \mathrm{msec}$ (Efron 1970a, 1970b, 1970c), the model predicts that the magnitude of the micropattern effect should (1) increase regularly with ISIs between 0 and $100 \mathrm{msec}$, (2) reach its maximum for ISIs between 100 and $200 \mathrm{msec}$, and (3) remain at that maximum level for ISIs greater than $200 \mathrm{msec}$.

\section{METHOD}

\section{Stimulus Parameters and Instrumentation}

The spatial arrangement of the stimulus is illustrated in Figure 2A. The 1-deg circular standard (S) and matching (M) micropattern stimuli were presented superimposed on the continuously present 5-deg neutral background field (BF). These stimuli were presented in Maxwellian view through a 2-mm-diam artificial pupil. The retinal illuminance of the stimuli was as follows: $B F=$ 10 trolands, $S=88$ trolands, and $M=123$ trolands. (The matching stimulus, $M$, was set to a higher retinal illuminance to compensate for a reduction in apparent brightness that occurred when the relative intensity of the trailing stimulus element was reduced to obtain the color match.)

The temporal parameters of the micropattern stimuli, $M$ and $S$, are illustrated in Figure 2B. The timing was done with a digital logic system (Iconix 6257-6010-6171). The light beams were switched on and off by means of shutter vanes mounted on the cones of Realistic 5A9 speaker coils driven by dc amplifiers; switching time from off to full intensity or the reverse was less than $1 \mathrm{msec}$. Each of the micropatterns consists of consecutive 10 -msec red (Wratten No. 29) and green (Wratten No. 99) elements. The micropatterns were presented repetitively, with the interstimulus interval (ISI) under the control of the experimenter. The order of the elements within the $S$ and $M$ micropatterns was always opposite; both orders were used.

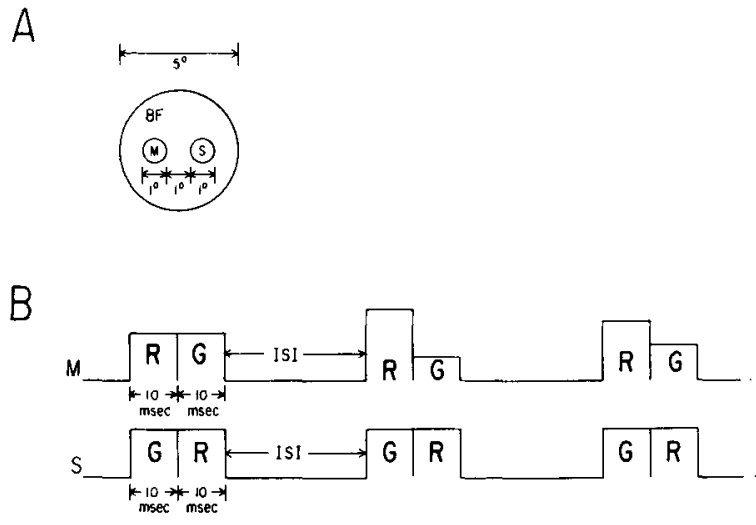

Figure 2. The repeating micropattern stimulus configuration. (A) The spatial arrangement: a 5-deg neutral background field upon which two 1-deg micropattern stimuli (M and S) were superimposed. (B) The temporal parameters of the micropattern stimuli: 10-msec red and green flashes presented in opposite orders in the standard (S) and matching (M) stimuli. The subject controlled the relative luminance of the flashes in $\mathbf{M}$. For each match setting, the interstimulus interval (ISI) was set by the experimenter to one of 11 values between 0 and 500 msec.

\section{Psychophysics}

For a given ISI and element order, the subject adjusted the proportion of the intensity in the red and green elements in the matching stimulus $(\mathrm{M})$ by means of a single rotary control. There was no time limit on a match setting; the subject indicated when the match was finished. Each experimental session consisted of one match setting for each of 22 stimulus conditions-11 ISIs (0, 20, $40,60,80,100,130,160,200,300,500 \mathrm{msec}$ ) for each element order. Twenty such sessions were run with each of four subjects. Within each of these 20 sessions, the 22 stimulus conditions were run in a different pseudorandom order. Two of the four subjects were two of the authors (B.Y. and H.M.); one of the subjects (D.N.M.) was totally unaware of the purpose of the experiment.

\section{RESULTS}

The results for each of the four subjects are given in one panel of Figure 3. Each point represents an average of $\mathbf{2 0}$ match settings; the error bars indicate $\pm 1 \mathrm{SD}$. The position of the neutral density wedges in the matching beams is plotted on the ordinate, and the interstimulus interval (ISI) is plotted on the abscissa. The sign of the wedge position value indicates whether the matching stimulus contained more red $(+)$ or more green $(-)$ component than the standard stimulus.

In essence, the two curves of each panel represent two repetitions of the experiment: for the upper curve, the subject adjusted the relative luminance of the elements of the red-green micropattern to match the standard green-red micropattern (the stimulus condition illustrated in Figure 2), and for the lower curve, the subject adjusted the relative luminance of the elements of the green-red micropattern to match the standard red-green micropattern. The only point common to the two curves, 0 -msec ISI, actually represents two independently measured points, each of 

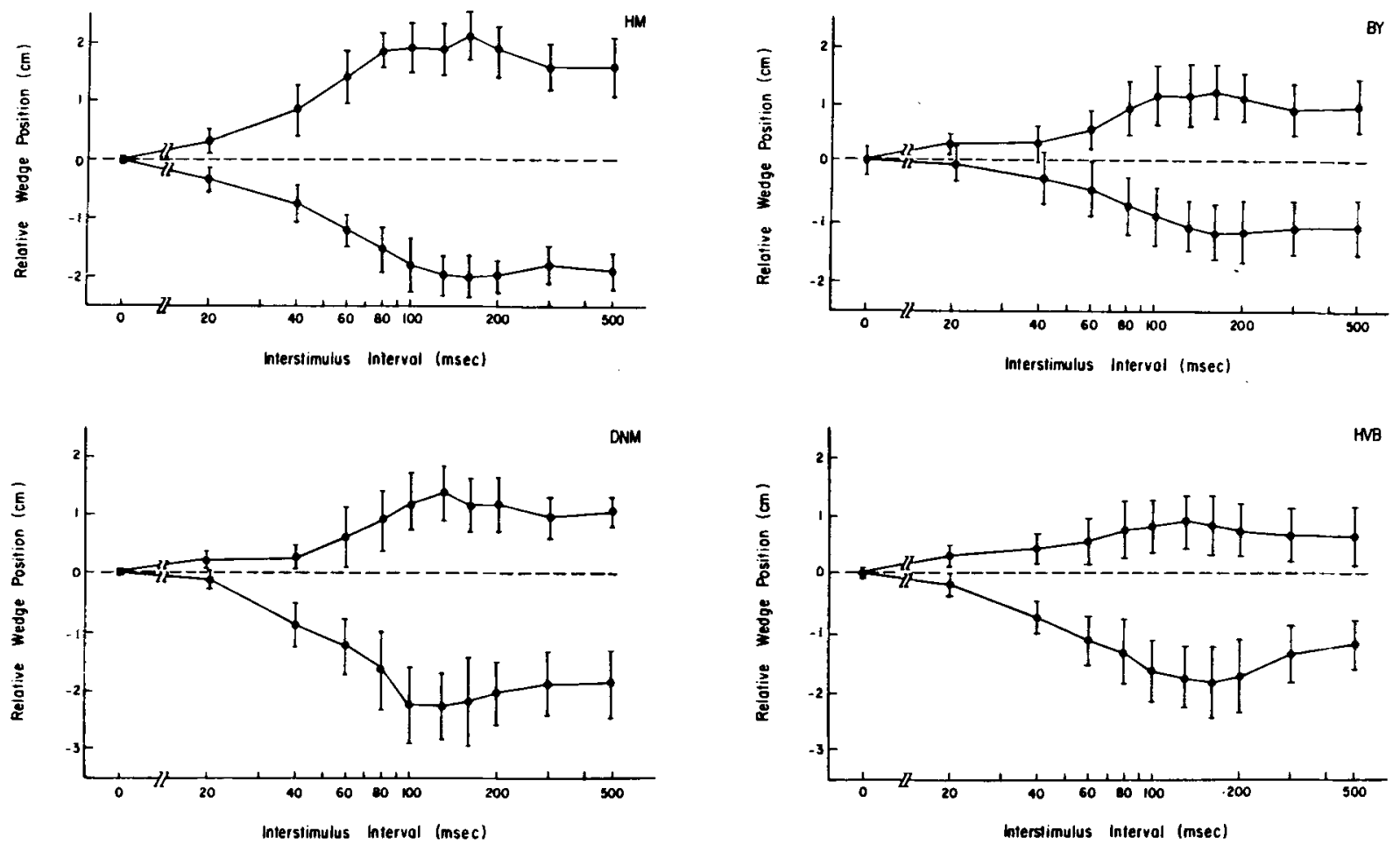

Figure 3. The results for each of the four subjects. The relative position of the neutral-density wedges in the two beams of the matching stimulus is plotted as a function of interstimulus interval. Positive wedge positions indicate more red light in the matching stimulus, and negative values indicate more green. The upper curve for each subject was measured for the green-red standard micropattern, and the lower curve for each was measured for the red-green standard. Each point is a mean of 20 measures; the error bars indicate $\pm 1 \mathrm{SD}$ for that point.

which logically belongs to one of these two curves, depending on the colors of the starting elements of the standard and matching stimuli. Each panel also includes a horizontal dashed line drawn through the 0 -msec ISI point to serve as a reference. The extent to which a point deviates from this line indicates the magnitude of the micropattern effect.

Let us first consider the results for Subject H.M. A micropattern effect occurred at every nonzero value of ISI tested; furthermore, the magnitude of the effect varies in a regular way with ISI. Between 0 and $100 \mathrm{msec}$, the data points on the two curves diverge from the dashed line, indicating that the magnitude of the micropattern effect increased monotonically as the ISI increased from 0 through $100 \mathrm{msec}$. At ISIs greater than $100 \mathrm{msec}$, however, the data points seem to remain at about the same distance from the dashed line, indicating little or no further change in the micropattern effect at longer ISIs.

The results for the other three subjects are very similar: The micropattern effect shows a monotonic increase in the ISI range from 0 to $100 \mathrm{msec}$ and little or no further increase beyond $200 \mathrm{msec}$.

Looking at all four graphs, however, we begin to see a pattern in the results at longer ISIs. The max- imum micropattern effects always occurred for ISIs below $200 \mathrm{msec}$ (100 msec for D.N.M.'s lower curve, $130 \mathrm{msec}$ for D.N.M.'s and H.V.B.'s upper curves, and $160 \mathrm{msec}$ for the other five curves). Micropattern effects at 200,300 , and 500 msec were always less than maximal, but even at $500 \mathrm{msec}$, the effect averaged $80 \%$ of its maximum. When considering the results at longer ISIs, it may also be important to note the subjective reports that stimuli with the longest ISIs seemed very different: the stimuli were not as bright and were experienced as intermittent. Although it is not clear what, if any, effects might be produced in conjunction with such subjective differences in the stimuli, these differences at least indicate that further experiments are needed before we can be sure how the micropattern effect varies at ISIs greater than $200 \mathrm{msec}$. It is also true that less variation in the micropattern effect at longer ISIs was found for the two individuals (H.M. and B.Y.) who had had the most previous experience as subjects in color-vision psychophysics.

In summary, the results demonstrate a monotonic increase in the magnitude of the micropattern effect as the time interval between micropatterns increases from 0 to about $100 \mathrm{msec}$. For intervals greater than 
$100 \mathrm{msec}$, the magnitude of the micropattern effect remained essentially constant or perhaps decreased slightly at and above $200 \mathrm{msec}$.

\section{DISCUSSION}

The results of these experiments clearly demonstrate that the dark interval immediately following a visual micropattern is critical in producing the micropattern effect. This had to be true at least in the trivial sense that the dark interval is necessary merely to define the stimulus: red-green and green-red repeating micropatterns with 0 -msec ISI are essentially identically regular alternations of red and green elements. But, given the fact that some dark interval is needed, what determines how the micropattern effect varies with ISI?

Before considering the visible persistence model described in the introduction of this report, it would be useful to consider briefly two other very simple possible explanations of the micropattern effect: (1) retroactive masking or inhibition of the first element by the second element, and (2) proactive enhancement or facilitation of the second element by the first element. A retroactive masking explanation has one problem that must be overcome: With simple uniform circular stimuli such as the micropattern elements used here, proactive masking is stronger than retroactive masking (Crawford, 1947). If proactive masking dominated in the micropattern stimulus, this would produce an effect that was the reverse of that found-the first element would mask the second, and the color of the leading element would be predominant in the color of the micropattern.

There is one form of retroactive masking that is greater than its proactive counterpart-metacontrastbut other properties of metacontrast seem to make it unsatisfactory as an explanation for the repeating micropattern results (Figure 3). Metacontrast masking is maximal at target-to-mask intervals greater than zero. Assuming that metacontrast masking occurs only between adjacent elements, there should be an ISI corresponding to the interval of maximum masking, at which the leading element of one micropattern masks the trailing element of the previous micropattern to a much greater extent than that trailing element is able to mask its own preceding element. At this ISI, the color of the leading element would dominate-the reverse of the micropattern effect. Since no results showing a reversed micropattern effect were obtained for any subject at any ISI, metacontrast-like interactions must be rejected as an explanation for the micropattern effect.

A possible proactive enhancement explanation is more difficult to formulate in any specific way. Although successive color-contrast effects are known for longer, more intense stimuli, little is known even of the existence of such a proactive enhancement for brief, moderate-intensity stimuli such as the micropattern elements used in this experiment. Unless more specific information about proactive facilitation is obtained, no useful proactive facilitation model for the micropattern effect can be formulated.

As described earlier in this report, the visiblepersistence model for the micropattern effect does make a specific prediction concerning the variation of the micropattern effect with ISI: The effect should increase monotonically with ISI between 0 and approximately $100 \mathrm{msec}$ and should remain constant with increases in ISI beyond that range. The results presented in Figure 3 are in accord with this prediction.

If there is any discrepancy between the results and this major prediction, that discrepancy occurs only at ISIs greater than $200 \mathrm{msec}$ : the maximum micropattern effects occurred for ISIs between 100 and $200 \mathrm{msec}$, and the magnitude of the effect was generally slightly less between 200 and 500 msec. Although we made no predictions of this apparently secondorder variation of the magnitude of the micropattern effect with ISI, it is clear in retrospect that this result is consistent with the visible persistence model in the following way: At the longest ISIs, the visual system's response to one micropattern should be completely finished when the next micropattern stimulus occurs. Under these conditions, the maximal possible response to the leading element shculd be generated. At intermediate ISIs, where the visible persistence of one micropattern is still present when the visual system is beginning to respond to the leading element of the next micropattern, a slightly less than maximal possible response to that leading element might be generated. Such a slight reduction in the response to the leading stimulus element would be seen in the results as an increase in the magnitude of the micropattern effect at the ISIs corresponding to the limits of the visible persistence, that is, in the range of 100 to $200 \mathrm{msec}$. Thus, it is clear that the visible persistence model for the micropattern effect can be used to explain not only the overall form of the results, but also the slight enhancement of the micropattern effect seen at intermediate ISIs.

One essential assumption, implicit in the visible persistence model for the micropattern effect, should be stated explicitly. The model requires that the information processing that determines the perceived color of a brief stimulus continue throughout the interval of the visible persistence. In this context, persistence cannot be conceived of as a mechanism for maintaining the visibility of a completed percept so that higher level cognitive information can be extracted from it-persistence is not a postperceptual "sample and hold" mechanism. Even such a relatively simple perceptual attribute as color depends 
upon the extent to which the visible persistence is permitted to run its full course. This assumption about visible persistence creates no difficulties for our model of the micropattern effect, since this assumption is consistent with at least one major theory for persistence-Sakitt's (1976) receptor theory. Of course, neither our model nor our results require that persistence occur in the very first stage of the visual system, but merely that it occur prior to the determination of perceived color.

\section{REFERENCES}

Crawrord, B. H. Visual adaptation in relation to brief conditioning stimuli. Proceedings of the Royal Society (London), 1947, 134B, 283-300.

Colthenrt, M. Iconic memory and visible persistence. Perception \& Psychophysics, 1980, 27, 183-228.
Erron, R. Effect of stimulus duration on perceptual onset and offset latencies. Perception \& Psychophysics, 1970, 8, 231-234. (a)

EFron, R. The minimum duration of perception. Neuropsychologia, 1970, 8, 57-63. (b)

Erron, $\mathbf{R}$. The relationship between the duration of a stimulus and the duration of a perception. Neuropsychologia, 1970, 8 , 37.55. (c)

Efron, R. Conservation of temporal information by perceptual systems. Perception \& Psychophysics, 1973, 14, 518-530.

Lono, G. M. Iconic memory: A review and critique of the study of short-term visual storage. Psychological Bulletin, 1980, 88, 785-820.

Sakitr, B. Iconic memory. Psychological Review, 1976, 83, 257-276.

Yund, E. W., \& EFron, R. Dichoptic and dichotic micropattern discrimination. Perception \& Psychophysics, 1974, 15, 383-390.

(Manuscript received February 3, 1983;

revision accepted for publication April 5, 1983.) 